\title{
The Effect of Acupressure on Anxiety and Depression Patients With ESRD Who are Undergoing Hemodialysis
}

\author{
* $1^{\text {st }}$ Made Suandika \\ School of Nursing \\ Universitas Harapan Bangsa \\ Purwokerto, Indonesia \\ gelansatra@gmail.com \\ $4^{\text {th }}$ Yun-Fang Tsai \\ School of Nursing College of Medicine \\ Chang Gung \\ University Tao-Yuan \\ Tao-yuan, Taiwan \\ yftsai@mail.cgu.edu.tw \\ $7^{\text {th }}$ Mariah Ulfah \\ School of Nursing \\ Universitas Harapan Bangsa \\ Purwokerto, Indonesia \\ mariahulfah@uhb.ac.id
}

\author{
$2^{\text {nd }}$ Woung-Ru Tang \\ School of Nursing College of Medicine \\ Chang Gung University R.O.C Taiwan \\ Tao-yuan, Taiwan \\ wtang@mail.cgu.edu.tw \\ $5^{\text {th }}$ Li-Chueh Weng \\ School of Nursing College of Medicine \\ Chang Gung \\ University Tao-Yuan \\ Tao-Yuan, Taiwan \\ ax2488@mail.cgu.edu.tw
}

\author{
$3^{\text {rd }}$ Ji-Tseng Fang \\ Department of Nephrology \\ Chang Gung Memorial Hospital \\ Tao-Yuan, Taiwan \\ fangjits@cgmh.org.tw \\ $6^{\text {th }}$ Pei-Kwei Tsai \\ School of Nursing College of Medicine \\ Chang Gung \\ University Tao-Yuan \\ Tao-yuan, Taiwan \\ tsay@mail.cgu.edu.tw \\ $8^{\text {th }}$ Linda Yanti \\ School of Nursing \\ School of Midwifery College of \\ Medicine \\ Universitas Harapan Bangsa \\ Purwokerto, Indonesia \\ mariahulfah@uhb.ac.id
}

\begin{abstract}
Anxiety and depression are very common symptoms in End Stage Renal Disease (ESRD) and acupressure a noninvasive procedure to promote health and good impact to quality of life (QoL) in hemodialysis (HD) patients. This study aimed to investigate the effect of acupressure on the anxiety and depression patients with ESRD who underwent HD. A experimental study with single blind between two groups was conducted at dialysis center in Central Java Province, Indonesia. Subjects with ESRD who undergoing HD were randomly divided into intervention group who received acupressure at K1, ST36, and SP6 acupoint, while the control group received sham acupressure. The both of the groups received treatment three time a week during for four week dialysis. Anxiety and depression was assessed by using Hospital Anxiety and Depression Scale (HADS). For data analysis was used t-test and SPSS software version 22 was used. 96 patients were enrolled and included either in experimental or control group. Acupressure induced significant improvement in anxiety $\&$ depression in treated patients compared to control (HADS: 7.653 vs 14.968; p-value: 0.000). Intragroup analysis showed that both groups experienced improvement in only experimental group experienced marked improvement in anxiety and depression. Acupressure could significantly and independently improve anxiety \& depression in ESRD patients undergoing HD.
\end{abstract}

Keywords-Hemodialysis, acupressure, anxiety, depression

\section{INTRODUCTION}

End Stage Renal Disease (ESRD) is a progressive and irreversible destruction of kidney function where the body's ability to maintain metabolism and water and electrolyte balance is removed $[1,2]$. in the United States, as many as $16.8 \%$ of the country's population suffers from ESRD and the number of patients suffering from ESRD is estimated at 3.346 .000 or more as of the end of 2014 [3, 4]. The most common treatment method used for ESRD patients is hemodialysis [5, 6]. Patients undergoing hemodialysis suffer from the limitations of these various factors which will result in the incidence and prevalence of mental disorders in ESRD patients. According to various studies conducted, one of the common psychological symptoms in these patients is anxiety and depression whose prevalence has been reported to range from 40 to $92 \%$ [7]. Anxiety is called uncertainty in feelings, fears, and panic [7-9]. Anxiety prevents individuals from following a recommended diet and treatment and has a negative effect on self-care and the treatment results received [2].

Anxiety treatment is done in two methods pharmacology and non-pharmacology. The general method, which uses chemical drugs for hemodialysis patients, is not included as one of the nurses' responsibilities and will result in increased risk for the patient, and this calls for adopting other necessary steps [7-9]. One of the most common non-drug methods is alternative medicine [10]. Alternative treatments include acupuncture, acupressure, massage therapy, aromatherapy, yoga, energy medicines[8] Among the approaches mentioned above, acupressure is one of the most popular methods to improve a patient's mental status.

Acupressure is one of the popular methods in traditional Chinese medicine where acupuncture points are stimulated by fingers by pressing and rubbing movements [11-13]. This traditional is used without using drugs or other chemicals. In addition, acupressure is cheap, safe, and has the fewest complications. In acupressure, pressure stimulation is carried out by giving massages to specific points of meridian 
energy[14]. Correcting the flow of energy will produce an appropriate response in various organs and glands. This method works by stimulating the secretion of neurotransmitters and adrenocorticotropic hormones through endorphin-mediated mechanisms [15].

Acupressure and energy balance are used to relieve pain, reduce muscle contraction, improve blood circulation status and performance of vital signs, and reduce anxiety symptoms (between 30 seconds to 5 minutes and even 20 minutes each time). Regarding its effect toward anxiety and depression, various studies have proved the effectiveness of acupressure on depression management using different acupoints. Tsay and colleagues reported that acupressure at 4 acupoints significantly improved depression in ESRD patients [16, 17]. Despite reporting improvement in psychological condition, the psychological part was assessed measurement and not using different tools. Therefore, this study aimed to investigate the efficacy of acupressure toward the level of anxiety \& depression and in patients with ESRD who underwent HD.

\section{RESEARCH METHOD}

Study design--- Our study is the part of the larger study. This study used single-blinded two-group experimental design, involving ESRD patients who underwent regular HD. Subjects were divided into two groups namely intervention group that received acupressure in the true acupoints, while the control group received sham acupressure. All ESRD patients who underwent HD in Central Java were involved as study population. The inclusion criteria included age > 18 years old, had been on HD for at least 3 months, fatigue level $\geqq 4$, the absence of wound on extremities, did not receive any complementary treatments for the past 3 months, and willing to participate by filling out questionnaires. Those with major depression or psychiatric disorders, wound or amputation on lower extremities, rheumatoid arthritis, or fracture of lower extremity were excluded.

Setting and samples----Acupressure was conducted 3 times per week for 4 weeks and a post test Patients who fulfilled the inclusion criteria were randomly assigned into two groups. Group 1, the experimental group, received acupressure at true acupoints (K1, ST36, and SP6), while group 2 (control) received acupressure at the sham acupoints. All of the acupressure in both groups were done only by the researchers. Acupressure was performed within the first 2 hours of HD by applying consistent pressure at each acupoint for 3 minutes with the rate of two rotations per second bilaterally.

Ethical considerations--- This study was approved by Jenderal Soedirman University from the purwokerto research ethics committee, through the ethics approval number: 3101 / KEPK / VII / 2019. Researchers have ensured that all participants understood the questionnaire and were able to communicate with researchers without the problem of language informed consent had been carried out for all subjects and approved. Finally, complete data from 96 patients were collected for statistical analysis.

Measurement of Anxiety and Depression.--Hospital Anxiety and Depression Scale (HADS) is a valid, reliable, and easy tool for doctors to identify and measure anxiety and depression[18]. HADS consists of 14 items which are divided into 2 subscales to assess anxiety (7 items) and depression (7 items). Subjects classified each item in 4 rating scales from 0 (not at all) to 3 (very often) with a higher value indicating a higher likelihood of anxiety / depression. HADS scores can be classified as follows: severe anxiety (16-21), moderate 11-15 anxiety (11-15); mild anxiety (8$10)$; and there is no anxiety or depression $(\leqslant 7)[18]$.

Data Analysis ----- All analyses were carried out using SPSS version 22. All data were compiled and compared between experimental and control group. Independent t-test was used to compare numerical data and chi-square was used to compare nominal data. Intragroup analysis was also conducted (using paired sample t-test) to visualize the improvement between pre- and postintervention within each group. A P-value of $<0.05$ was considered statistically significant.

\section{FINDING AND DISCUSSION}

Initially, 106 participants were selected according to the sampling criteria but 10 participants were excluded due to the following reasons: 3 respondents disagreed in the intervention for 1 week (changed in schedule), 3 patients were accepted, 1 patient refused to complete in the entire program, and 3 respondents issued HIV / AIDS. Finally, 96 study subjects were divided into experimental $(n=49)$ and control groups $(n=47)$.

Then, the level of baseline data that was agreed \& corrected in the groups that were approved and compared (Table 1). According to HADS, it seems that this group has moderate HADS control, both groups have resolution and moderate (HADS: control vs experimental: 14.681 vs 13.592; p-value: 0.094). All measurements and subcomponents are comparable between the two groups.

TABLE I. THE INDEPENDENT T-TEST ANALYSIS OF THE ANXIETY \& DEPRESSION A SCORE BETWEEN THE GROUPS AT BASELINE IN THE PRE INTERVENTION $(\mathrm{N}=96)$

Table 1 The Independent t-test analysis of the, Anxiety \& depression a score between the

\begin{tabular}{|c|c|c|c|c|c|c|}
\hline \multirow{2}{*}{ Variables } & \multicolumn{2}{|c|}{$\begin{array}{l}\text { Experimental Group } \\
(\mathrm{N}=49)\end{array}$} & \multicolumn{2}{|c|}{ Control Group ( $\mathrm{N}=47$ ) } & \multirow{2}{*}{$\mathrm{t}$} & \multirow{2}{*}{$\mathrm{p}$ value } \\
\hline & Mean & SD & Mean & SD & & \\
\hline Pre Anxiety & 13.980 & 2.940 & 14.936 & 5.227 & -1.099 & 0.275 \\
\hline Pre Depression & 13.204 & 2.264 & 14.426 & 3.894 & -1.869 & 0.066 \\
\hline Pre HADS Score & 13.592 & 1.850 & 14.681 & 4.006 & -1.698 & 0.094 \\
\hline
\end{tabular}

* HADS: Hospital Anxiety and Depression Scale

After 4 weeks of acupressure treatment, HADS scores returned and compared between groups (Table 2). Overall, the experimental group had a significantly lower HADS value compared to the control group. However, changes in HADS scores are clearer. The average of the two control variables was significantly lower and higher than HADS: trial vs control: 7.653 vs 14.968 ; p-value: 0.000 ). 
TABLE II. THE INDEPENDENT T-TEST OF THE ANXIETY \& DEPRESSION SCORE BETWEEN THE GROUPS AT BASELINEIN THE POST INTERVENTION $(\mathrm{N}=96)$

\begin{tabular}{|c|c|c|c|c|c|c|}
\hline \multirow{2}{*}{ Variables } & \multicolumn{2}{|c|}{$\begin{array}{l}\text { Experimental } \\
\text { Group }(\mathrm{N}=49)\end{array}$} & \multicolumn{2}{|c|}{$\begin{array}{l}\text { Control Group } \\
(\mathrm{N}=47)\end{array}$} & \multirow{2}{*}{$t$} & \multirow{2}{*}{$p$ valuc } \\
\hline & Mean & SD & Mean & SD & & \\
\hline Post Anxiety & 7.449 & 2.777 & 15.000 & 4.974 & -9.132 & 0.000 \\
\hline Post Depression & 7.857 & 2.198 & 14.936 & 4.229 & -10.226 & 0.000 \\
\hline Post HADS Score & 7.653 & 1.910 & 14.968 & 4.276 & -10.744 & 0.000 \\
\hline
\end{tabular}

HADS: Hospital Anxiety and Depression Scale

This change shows that increasing the variables in the experimental group compared to controls. To visualize changes in depression / conversion in the second group, intra-group analysis by comparing the HADS score values was carried out (Table 2).

Anxiety \& depression are the most common mental health problems in HD patients[19], and these issues can pose negative impacts on ESRD patients' life quality (Qol) $[19,20]$. The results of our studies have found that acupressure significantly reduces the anxiety \& depression score of the subjects in the intervention group compared to those in the control group (sham group). The major finding of our studies is congruent with those of previous studies testing the effectiveness of acupressure on anxiety \& depression in ESRD population [16, 17, 19, 20]

The results of this study are similar to those of Mischoulon et al who assess the effectiveness and safety of acupuncture monotherapy in major depressive disorders. Assessment using the HADS score gives an acupressure response response> 50\%; same as treatment with antidepressants. Qu et al[21] conducted a combination acupressure study in major depressive disorder [21, 22]. The results showed that after 6 weeks of acupressure therapy, clinical response was better in the manual acupressure group [21]. Wang et al (2016) conducted a meta-analysis of the effect of acupressure on depressive disorders [23, 24]. The meta-analysis included 8 studies with 477 depressed patients and 256 patients treated with acupressure and from the metaanalysis it was found that acupressure was proven to reduce depressive symptoms as indicated by a decrease in HADS score at the end of therapy $[6,25]$.

The mechanism of action of acupressure in depressive disorders is still not well known [26]. Acupressure stimulation is closely related to the increase in monoamine neurotransmitters, especially serotonin and norepinephrine [27-29]. Stimulation of acupressure both manual and electro acupuncture can facilitate the production of serotonin in the brainstem and modulate the activity of norepinephrine at the locus seruleus, hypothalamus, amygdala and prefrontal cortex to facilitate the production of serotonin in the brain stem and modulate the activity of norepinephrine, hypothalamus, amygdala and prefrontal cortex to facilitate mood regulation [27-29]. Acupressure can also increase the availability of neurotransmitters in nerve synapses thereby increasing interaction with post synapse cells [30].

Acupressure in ST36 acupoint can increase the availability of neurotransmitters such as serotonin by accelerating the synthesis and use of neurotransmitters in the central nervous system [31]. In addition, acupressure in ST36 can also strengthen the antidepressant effect by increasing serotonergic and or nor-edrenergic activity in synapses, thereby producing a combination of acupuncture with the effect of acupuncture with the effect antidepressant medication [32]. Repeated anxiety and depression that lasts a long time will reduce the sensitivity of Cortisol Releasing Hormon $(\mathrm{CRH})$ resulting in HPA axis disruption in the form of the inability of cortisol to suppress CRH secretion [33]. Several study shown acupressure significantly reduced symptoms of depression and anxiety by increasing the expression of Neuropeptide Y (NPY) in the hypothalamus, NPY is a neuropeptide in the hypothalamus which decreases the activity of the HPA axis which plays an important role in anxiety and depression [34-40].

Acupressure is synergistic with antidepressant drugs to maintain serotonin levels and nor-adrenaline activity in synaptic gaps, resulting in a relatively safer action with serotonin and nor-epinephrine reuptake inhibitors (SNRIs) [41-43].

The superiority of acupressure in ST36 compared to antidepressant drugs is that they are relatively safer with minimal side effects of acupressure with side effects [44], such as hematoma and bleeding at the massage site so that it is safe to apply [45]. The drawback is that patients must make repeated visits to undergo acupressure therapy [46]

Acupressure has been proved to be potent enough to relieve anxiety \& depression in patients with various type of cancer who undergo chemotherapy [47, 48]. Tang et al., reported that acupressure significantly improved anxiety \& depression in lung cancer patients with consistent improvement between chemotherapy cycles [47]. However, no effects were observed in HADS. Similar findings reported by $\mathrm{Qu}$ et al[21] who showed that 6 weeks of acupressure therapy was effective in alleviating depression in major depressive disorder. Finally, a meta-analysis showed that acupressure is an effective approach in relieving anxiety and depression in cancer patients[41].

However, it also noted that definitive conclusion could not be drawn due to small number of studies included, relatively small number of subjects per study and short follow up time. Physiologically, there are only limited evidences about how acupressure works. Several studies showed that acupressure primarily stimulates spino-thalamocortico-limbic system, inducing endorphin release which enhances comfortable feeling [16, 49-52]. Furthermore, there are also evidences of increased dopamine and serotonin release in the synaptic cleft which enhance motivation and induce muscle relaxation[53].

\section{LIMITATION}

This is study was short follow up period. Future research must include longitudinal studies of acupressure on anxiety for ESRD patient in the clinical setting and continued at the home of the patients.

\section{CONCLUSION}

The findings from our study and previous studies demonstrate the effectiveness of acupressure on anxiety \& depression management. According to the results of this 
study, it can be concluded that pre-dialysis, 4 weeks acupressure therapy of at K1, ST36, and SP6 acupoints significantly reduced anxiety \& depression in patients with ESRD. Therefore, we strongly recommend that health care providers and patients should be informed of the benefits of acupressure on depression management

These findings add new evidences that support the potential application of acupressure in ESRD patients as nonpharmacology alternative therapy to alleviate psychological disorders. It would also reduce renal burden and additional risk of administering pharmacological agents to patients with reduced renal function. Due to the easiness of its application, acupressure also versatile enough to be applied not only by medical personnel but also patient's family or guardian with proper training.

\section{ACKNOWLEDGMENT}

The author tanks to for Dr Fang to teach the basic of acupressure techniques. We appreciate very much to helpful support given by the nephrologist, nurse manager and staff nurse hemodialysis center.

\section{REFERENCES}

[1] K. S. Astroth, C. L. Russell, and J. L. Welch, "Non-pharmaceutical fatigue interventions in adults receiving hemodialysis: a systematic review," (in eng), Nephrol Nurs J, vol. 40, no. 5, pp. 407-27; quiz 428, Sep-Oct 2013.

[2] L. S. Wieland and N. Santesso, "A Summary of a Cochrane Review: Acupuncture or acupressure for induction of labour," (in eng), Eur J Integr Med, vol. 17, pp. 141-142, Jan 2018.

[3] USRDS, "2018 USRDS Annual Data Report: Executive Summary," 2018 USRDS Annual Data Report, 2018.

[4] USRDS, "Chapter 1: Incidence, Prevalence,Patient Characteristics, and Treatment Modalities ESRD in US," vol. 2, 2018.

[5] J. L. Wainright, A. M. Robinson, A. R. Wilk, D. K. Klassen, W. S Cherikh, and D. E. Stewart, "Risk of ESRD in prior living kidney donors," (in eng), Am J Transplant, vol. 18, no. 5, pp. 1129-1139, May 2018.

[6] R. Wang et al., "Poor sleep and reduced quality of life were associated with symptom distress in patients receiving maintenance hemodialysis," (in eng), Health Qual Life Outcomes, vol. 14, no. 1, p. 125, Sep 082016.

[7] Y. S. Zeng, C. Wang, K. E. Ward, and A. L. Hume, "Complementary and Alternative Medicine in Hospice and Palliative Care: A Systematic Review," (in eng), J Pain Symptom Manage, vol. 56, no. 5, pp. 781-794.e4, Nov 2018.

[8] B. Yang et al., "Non-pharmacological interventions for improving sleep quality in patients on dialysis: systematic review and metaanalysis," (in eng), Sleep Med Rev, vol. 23, pp. 68-82, Oct 2015.

[9] X. Zhou et al., "The quality of life and associated factors in patients on maintenance hemodialysis - a multicenter study in Shanxi province," (in eng), Ren Fail, vol. 39, no. 1, pp. 707-711, Nov 2017.

[10] M. Weiss, T. Mettang, U. Tschulena, J. Passlick-Deetjen, and E. Weisshaar, "Prevalence of chronic itch and associated factors in haemodialysis patients: a representative cross-sectional study," (in eng), Acta Derm Venereol, vol. 95, no. 7, pp. 816-21, Sep 2015.

[11] G. J. McDougall, "Research review: the effect of acupressure with massage on fatigue and depression in patients with end-stage renal disease," (in eng), Geriatr Nurs, vol. 26, no. 3, pp. 164-5, May-Jun 2005

[12] W. E. Mehling et al., "Symptom management with massage and acupuncture in postoperative cancer patients: a randomized controlled trial," J Pain Symptom Manage, vol. 33, no. 3, pp. 258-66, Mar 2007.

[13] R. Mehrotra, O. Devuyst, S. J. Davies, and D. W. Johnson, "The Current State of Peritoneal Dialysis," (in eng), J Am Soc Nephrol, vol. 27, no. 11, pp. 3238-3252, Nov 2016.
[14] E. Patterson, Y. W. Wan, and S. Sidani, "Nonpharmacological nursing interventions for the management of patient fatigue: a literature review," (in eng), J Clin Nurs, vol. 22, no. 19-20, pp. 266878, Oct 2013.

[15] M. Motalebi and B. Einollahi, "Remarks about the study of quality of life and sleep in hemodialysis patients," (in eng), Saudi J Kidney Dis Transpl, vol. 25, no. 4, pp. 883-4, Jul 2014.

[16] S. L. Tsay, "Acupressure and fatigue in patients with end-stage renal disease-a randomized controlled trial," (in eng), Int J Nurs Stud, vol. 41, no. 1, pp. 99-106, Jan 2004.

[17] S. L. Tsay, Y. C. Cho, and M. L. Chen, "Acupressure and Transcutaneous Electrical Acupoint Stimulation in improving fatigue, sleep quality and depression in hemodialysis patients," (in eng), Am J Chin Med, vol. 32, no. 3, pp. 407-16, 2004

[18] A. S. Zigmond and R. P. Snaith, "The hospital anxiety and depression scale," (in eng), Acta Psychiatr Scand, vol. 67, no. 6, pp. 361-70, Jun 1983.

[19] N. T. Hmwe, P. Subramanian, L. P. Tan, and W. K. Chong, "The effects of acupressure on depression, anxiety and stress in patients with hemodialysis: a randomized controlled trial," (in eng), Int J Nurs Stud, vol. 52, no. 2, pp. 509-18, Feb 2015.

[20] L. Kalani, H amidreza A ghababaeian, Narges M ajidipour, Milad A lasvand, Hadi B ahrami, and M. V. a. F akhri S abouhi, Mojgan M ortazavi, Jahangir E xir, "The effects of acupressure on severity of depression in hemodialysis patients: a randomized controlled Trial," Journal of Advanced Pharmacy Education \& Research, vol. 9, no. 52, pp. 67-72, 2019

[21] F. Qu et al., "Auricular acupressure reduces anxiety levels and improves outcomes of in vitro fertilization: a prospective, randomized and controlled study," (in eng), Sci Rep, vol. 4, p. 5028, May 222014.

[22] M. F. Pai, S. P. Hsu, S. Y. Yang, T. I. Ho, C. F. Lai, and Y. S. Peng, "Sleep disturbance in chronic hemodialysis patients: the impact of depression and anemia," (in eng), Ren Fail, vol. 29, no. 6, pp. 673-7, 2007.

[23] S. Y. Wang et al., "Factors related to fatigue in Chinese patients with end-stage renal disease receiving maintenance hemodialysis: a multicenter cross-sectional study," (in eng), Ren Fail, vol. 38, no. 3, pp. $442-50,2016$

[24] S. Y. Wang, X. Y. Zang, J. D. Liu, M. Cheng, Y. X. Shi, and Y. Zhao, "Indicators and correlates of psychological disturbance in Chinese patients receiving maintenance hemodialysis: a crosssectional study," (in eng), Int Urol Nephrol, vol. 47, no. 4, pp. 679-89, Apr 2015

[25] C. Y. Wei, T. C. Chung, S. C. Wu, C. F. Chung, and W. P. Wu, "The subjective sleep quality and heart rate variability in hemodialysis patients," (in eng), Ren Fail, vol. 33, no. 2, pp. 109-17, 2011.

[26] S. Williams et al., "Physical Activity and Sleep Patterns in Hemodialysis Patients in a Suburban Environment," (in eng), Blood Purif, vol. 43, no. 1-3, pp. 235-243, 2017.

[27] P. Delmas et al., "Effects on nurses' quality of working life and on patients' quality of life of an educational intervention to strengthen humanistic practice among hemodialysis nurses in Switzerland: a protocol for a mixed-methods cluster randomized controlled trial," BMC Nurs, vol. 17, p. 47, 2018.

[28] J. A. Dennis, A. Alazzeh, A. M. Kumfer, R. McDonald-Thomas, and A. N. Peiris, "The Association of Unreported Sleep Disturbances and Systemic Inflammation: Findings from the 2005-2008 NHANES," (in eng), Sleep Disord, vol. 2018, p. 5987064, 2018.

[29] F. Rahimi, S. Goli, N. Soltani, H. Rezaei, and Z. Amouzeshi, "Effects of Complementary Therapies on Labor Pain: A Literature Review," Modern Care Journal, vol. 15, no. 1, 2018

[30] J. Fleckenstein et al., "Acupuncture reduces the time from extubation to 'ready for discharge' from the post anaesthesia care unit: results from the randomised controlled AcuARP trial," (in eng), Sci Rep, vol. 8, no. 1, p. 15734, Oct 242018.

[31] T. W. Garcia, J. P. Veiga, L. D. Motta, F. J. Moura, and L. A. Casulari, "Depressed mood and poor quality of life in male patients with chronic renal failure undergoing hemodialysis," (in eng), Braz J Psychiatr, vol. 32, no. 4, pp. 369-74, Dec 2010.

[32] N. L. Garabed Eknoyan, et al, "KDIGO 2017 Clinical Practice Guideline Update for the Diagnosis, Evaluation, Prevention, and Treatment of Chronic Kidney Disease-Mineral and Bone Disorder 
(CKD-MBD)," (in english), OFFICIAL JOURNAL OF THE INTERNATIONAL SOCIETY OF NEPHROLOGY, vol. volume 7, no. issue 1, pp. 1-59, july 20172017.

[33] Y. Li et al., "Use acupuncture to relieve perimenopausal syndrome: study protocol of a randomized controlled trial," (in eng), Trials, vol. 15, p. 198, May 302014.

[34] S. Lee and H. Park, "The Effects of Auricular Acupressure on Smoking Cessation for Male College Students," (in eng), West J Nurs Res, vol. 39, no. 3, pp. 374-387, Mar 2017.

[35] D. E. Leaf and D. S. Goldfarb, "Interpretation and review of healthrelated quality of life data in CKD patients receiving treatment for anemia," (in eng), Kidney Int, vol. 75, no. 1, pp. 15-24, Jan 2009.

[36] Y. C. Learmonth, D. Dlugonski, L. A. Pilutti, B. M. Sandroff, R. Klaren, and R. W. Motl, "Psychometric properties of the Fatigue Severity Scale and the Modified Fatigue Impact Scale," J Neurol Sci, vol. 331, no. 1-2, pp. 102-7, Aug 152013

[37] A. Lee, S. K. Chan, and L. T. Fan, "Stimulation of the wrist acupuncture point PC6 for preventing postoperative nausea and vomiting," (in eng), Cochrane Database Syst Rev, no. 11, p. Cd003281, Nov 22015.

[38] E. J. Lee and S. K. Frazier, "The efficacy of acupressure for symptom management: a systematic review," (in eng), J Pain Symptom Manage, vol. 42, no. 4, pp. 589-603, Oct 2011.

[39] J. M. Lee et al., "Comparison of outcomes after treatment of in-stent restenosis using newer generation drug-eluting stents versus drugeluting balloon: Patient-level pooled analysis of Korean Multicenter in-Stent Restenosis Registry," (in eng), Int J Cardiol, vol. 230, pp. 181-190, Mar 12017.

[40] J. S. Lee, S. H. Hwang, B. C. Shin, and Y. M. Park, "Electrical stimulation of auricular acupressure for dry eye: A randomized controlled-clinical trial," (in eng), Chin J Integr Med, vol. 23, no. 11, pp. 822-828, Nov 2017.

[41] W. M. Ling, L. Y. Lui, W. K. So, and K. Chan, "Effects of acupuncture and acupressure on cancer-related fatigue: a systematic review," (in eng), Oncol Nurs Forum, vol. 41, no. 6, pp. 581-92, Nov 12014.

[42] K. M. Levett, C. A. Smith, H. G. Dahlen, and A. Bensoussan, "Acupuncture and acupressure for pain management in labour and birth: a critical narrative review of current systematic review evidence," (in eng), Complement Ther Med, vol. 22, no. 3, pp. 523-40, Jun 2014.
[43] G. W. Kang, I. H. Lee, K. S. Ahn, J. Lee, Y. Ji, and J. Woo, "Clinical and psychosocial factors predicting health-related quality of life in hemodialysis patients," (in eng), Hemodial Int, vol. 19, no. 3, pp. 43946, Jul 2015.

[44] Y. Liang, G. B. Lenon, and A. W. H. Yang, "Acupressure for respiratory allergic diseases: a systematic review of randomised controlled trials," (in eng), Acupunct Med, vol. 35, no. 6, pp. 413-420, Dec 2017.

[45] G. H. Lin, W. C. Chang, K. J. Chen, C. C. Tsai, S. Y. Hu, and L. L. Chen, "Effectiveness of Acupressure on the Taichong Acupoint in Lowering Blood Pressure in Patients with Hypertension: A Randomized Clinical Trial," (in eng), Evid Based Complement Alternat Med, vol. 2016, p. 1549658, 2016.

[46] B. Joshwa, D. C. Khakha, and S. Mahajan, "Fatigue and depression and sleep problems among hemodialysis patients in a tertiary care center," (in eng), Saudi J Kidney Dis Transpl, vol. 23, no. 4, pp. 72935, Jul 2012.

[47] W. R. Tang et al., "Effects of acupressure on fatigue of lung cancer patients undergoing chemotherapy: an experimental pilot study," (in eng), Complement Ther Med, vol. 22, no. 4, pp. 581-91, Aug 2014.

[48] A. G. Khanghah, M. S. Rizi, B. N. Nabi, M. Adib, and E. K. N. Leili, "Effects of Acupressure on Fatigue in Patients with Cancer Who Underwent Chemotherapy," J Acupunct Meridian Stud, vol. 12, no. 4, pp. 103-110, Aug 2019.

[49] R. E. Harris et al., "Using acupressure to modify alertness in the classroom: a single-blinded, randomized, cross-over trial," (in eng), $J$ Altern Complement Med, vol. 11, no. 4, pp. 673-9, Aug 2005.

[50] N. Paramita et al., "Validity and Reliability of the Indonesian Version of the Brief Fatigue Inventory in Cancer Patients," J Pain Symptom Manage, vol. 52, no. 5, pp. 744-751, Nov 2016.

[51] S. Li, D. Ye, G. Chen, and W. Xu, "Meta-Analysis of Comparison of Drug-Eluting Stents and Bare-Metal Stents in Patients on Dialysis," (in eng), Am J Cardiol, vol. 119, no. 8, pp. 1186-1192, Apr 152017.

[52] P. Mehta, V. Dhapte, S. Kadam, and V. Dhapte, "Contemporary acupressure therapy: Adroit cure for painless recovery of therapeutic ailments," (in eng), J Tradit Complement Med, vol. 7, no. 2, pp. 251263, Apr 2017.

[53] D. J. Wu, H. C. Dong, T. N. Tang, and S. F. Zhu, "Acupressure for insomnia: A protocol for systematic review and meta-analysis," (in eng), Medicine (Baltimore), vol. 97, no. 45, p. e13180, Nov 2018. 